\title{
How Often Are Patient-Important Outcomes Represented in Neonatal Randomized Controlled Trials? An Analysis of Cochrane Neonatal Reviews
}

\author{
Nai Ming Lai ${ }^{a}$ b Denise Yin Xian Leom ${ }^{c}$ Wen Li Chow ${ }^{d}$ Kee-Hsin Chen ${ }^{\text {e-g }}$ \\ Pu-Hong Lin ${ }^{f}$ Nathorn Chaiyakunapruk ${ }^{h}$ Colleen Ovelman ${ }^{i}$ Roger Solli,j

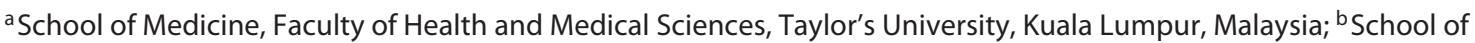 \\ Pharmacy, Monash University, Kuala Lumpur, Malaysia; ' Department of Emergency and Trauma, Labuan Hospital, \\ Labuan, Malaysia; ${ }^{d}$ Department of Paediatrics, Hospital Sultanah Nora Ismail, Batu Pahat, Batu Pahat, Malaysia; \\ ePost-Baccalaureate Program in Nursing, College of Nursing and Cochrane Taiwan, Taipei Medical University, Taipei, \\ Taiwan; ${ }^{f}$ Department of Nursing, Wan Fang Hospital, Taipei Medical University, Taipei, Taiwan; ${ }^{9}$ Evidence-Based \\ Knowledge Translation Center, Wan Fang Hospital, Taipei Medical University, Taipei, Taiwan; ${ }^{\text {h}}$ Department of \\ Pharmacotherapy, College of Pharmacy, University of Utah, Salt Lake City, UT, USA; 'Cochrane Neonatal, Burlington, \\ VT, USA; ' jDivision of Pediatrics - Neonatology, The University of Vermont Medical Center, Burlington, VT, USA
}

\section{Keywords}

Neonatal outcome - Randomized controlled trial ·

Patient-important outcomes

\begin{abstract}
Background: Research findings based on patient-important outcomes (PIOs) provide more useful conclusions than those that are based on surrogate outcomes. It is unclear to what extent PIOs are represented in neonatal randomized controlled trials (RCTs). Objectives: We determined the proportion of PIOs in neonatal RCTs included in Cochrane Neonatal reviews. Methods: We extracted up to 5 outcomes from each RCT included in Cochrane Neonatal reviews published until January 2018, with independent determination of PIOs among authors followed by a discussion leading to a consensus. We defined PIOs as outcomes that matter to patient care, such as clinical events or physiological or laboratory parameters that are widely used to guide management. $\boldsymbol{R e}$ sults: Among 6,832 outcomes extracted from 1,874 RCTs included in 276 reviews, 5,349 (78.3\%) were considered PIOs; 461 studies (24.5\%) included 5 or more PIOs, 1,278 (68.2\%)
\end{abstract}

included 1-4 PIOs, while 135 (7.2\%) had no PIO included. PIOs were observed more often among dichotomous than among continuous outcomes (94.9 vs. $61.5 \%$; RR: 1.54 ; $95 \%$ Cl: 1.50-1.58), and more among subjective than among objective outcomes (95.9 vs. 76.8\%; RR: 1.25; 95\% Cl: 1.22-1.28). Newer studies were more likely to have a greater number of PIOs (adjusted OR: 1.033 [95\% Cl: 1.025-1.041] with each publication year). Conclusions: The large and increasing representation of PIOs over the years suggests an improving awareness by neonatal trialists of the need to incorporate important outcomes in order to justify the utilization of resources. Further research should explore the reasons for non-inclusion or non-reporting of PIOs in a small proportion of RCTs.

(c) 2020 S. Karger AG, Basel

\section{Background}

A clinical trial that is useful for informing practice should have credible methodologies [1], good quality of reporting [2], and findings that are interpretable and ap- karger@karger.com

(C) 2020 S. Karger AG, Basel

www.karger.com/neo

Karger ${ }^{\prime}=$
Prof. Nai Ming Lai

School of Medicine, Faculty of Health and Medical Sciences

Taylor's University

Kuala Lumpur, Selangor Darul Ehsan 47500 (Malaysia)

lainm@ doctors.org.uk 
plicable. A study is more likely to be interpretable and applicable by having a representative population, an intervention strategy that is feasible and reproducible, comparators that are widely used in current practice, and outcomes that are relevant to health care providers and patients [3].

Findings of clinical research that incorporate patientimportant outcomes (PIOs) provide more trustworthy conclusions compared to those derived from surrogate outcomes such as laboratory parameters. The relevance or directness of the outcomes regarding patient care is also a factor in determining the certainty of evidence, which is a major consideration in the formulation of practice recommendations [4].

However, incorporating appropriate outcomes presents a challenge to researchers $[5,6]$. Problems in selection and reporting of outcomes in clinical trials include inconsistent reporting by the use of different outcome definitions, different evaluation tools, and different periods of assessment [7-9], as well as the use of evaluation tools that are not generally used in practice, evaluation of short-term outcomes when outcomes that matter are long term, and the use of surrogate outcomes rather than PIOs [10]. Moreover, the importance of outcomes varies between population groups and individual patients [9, 11].

A surrogate outcome is an outcome that is used to represent or predict the outcome of interest. It is usually a physiological or laboratory parameter, but sometimes it takes the form of a clinical finding [12]. A surrogate outcome is usually a continuous outcome that is objective and readily measurable - hence its popularity among researchers, as it enables a trial to be conducted with a comparatively smaller sample size, shorter duration, and lower cost compared to a trial that relies solely on major clinical outcomes [13]. However, the relationship between a surrogate outcome and the corresponding major clinical outcome of interest is often poor or unclear, and the use of surrogate outcomes may cause a misleading interpretation of findings, which in turn leads to inappropriate practice recommendations $[14,15]$. Examples of inappropriate use of surrogate outcomes that has caused harm include the use of a drug that reduced asymptomatic cardiac arrhythmia but increased fatal arrhythmias, and costly drugs that improved lipid profiles but made no difference in the risk of important cardiovascular events [16].

It has been recognized that PIOs are generally poorly represented in individual trials [17] and in systematic reviews $[7,18]$. To improve the incorporation of PIOs in clinical trials, several initiatives have been undertaken to provide researchers guidance on the selection and definition of outcomes. One notable example is the COMET (Core Outcome Measures in Effectiveness Trials) initiative, which was a major effort involving a large number of stakeholders that aimed to provide guidance to those undertaking clinical research on the set of major outcomes that should be incorporated in clinical trials of different conditions $[19,20]$. Pre-specification of PIOs as primary outcomes has also been made mandatory in Cochrane Systematic Reviews [21].

Neonatology is one of the most fertile fields in clinical research, where practice recommendations based on findings from RCTs conducted over six decades have improved the care of the newborn, especially the very premature and those with low birth weights $[22,23]$. As part of the COMET initiative, efforts to define a core outcome set in neonatal trials have been undertaken in the form of Core Outcomes in Neonatology (COIN). In this exercise, 12 outcomes were selected as the most important outcomes in neonatal care by 414 stakeholders from 25 counties that consisted of former patients, carers and caregivers, health professionals, and researchers. The outcomes selected were survival, sepsis, necrotizing enterocolitis, brain injury on imaging, retinopathy of prematurity, general gross motor and cognitive abilities, quality of life, adverse events, visual and hearing impairments, and bronchopulmonary dysplasia [24]. Although the initiative focused on important outcomes mainly for high-income countries, most of the outcomes identified are applicable globally. However, it was unclear to what extent PIOs such as those identified in the COIN initiative were represented in published neonatal RCTs.

In this study, we determined the proportion and characteristics of PIOs in neonatal RCTs that were included in Cochrane Neonatal reviews.

\section{Methods}

We conducted an analysis of the individual trials included in published Cochrane Neonatal reviews up to January 2018 (issue 1). We extracted relevant information from the "Characteristics of Included Studies" table in each Cochrane review. From studies that included more than 5 outcomes, we extracted 5 outcomes that we considered to be of the greatest importance to patients (usually the major clinical outcomes), due to constraints of time allocated to this project. This study was approved by Cochrane Neonatal, and used only published data from Cochrane Neonatal reviews.

We categorized the outcomes extracted according to the following characteristics: (1) type of data (dichotomous, continuous, or ordinal); (2) nature (subjective or objective); and (3) predominant method of assessment (clinical, radiological, carers'/caregivers' perception, physiological parameters [e.g., heart rate and re- 


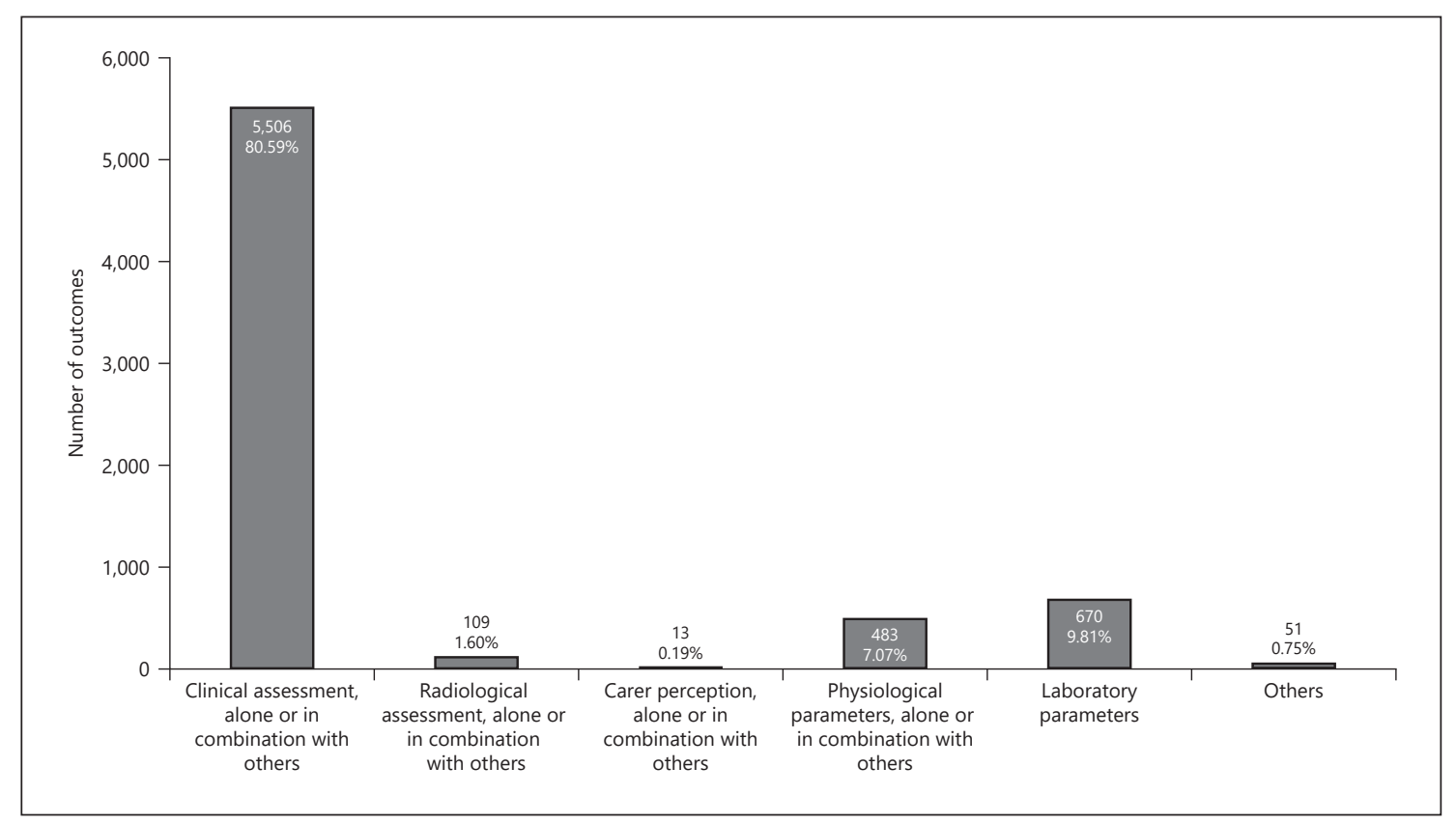

Fig. 1. Classification of outcomes according to the method of assessment.

spiratory rate, which are determined mainly at the bedside], or laboratory parameters, alone or in combination with other methods or with methods other than the 5 mentioned).

Following the main objective, we categorized the outcomes into "PIOs" and "non-PIOs." We defined PIOs as outcomes that patients value directly $[16,21,25,26]$. This included major clinical events (mortality and morbidities) as well as patients' or carers'/ caregivers' perception. For the purposes of this study, we considered certain surrogate outcomes that are used routinely to guide patient management as PIOs, and these included physiological or laboratory parameters such as oxygen requirement, hypotension, pain scale score, serum bilirubin, and serum glucose. We considered other physiological or laboratory parameters that were not used routinely to guide patient management as non-PIOs.

Two pairs of authors (D.Y.X.L. and W.L.C., as well as K.-H.C. and P.-H.L.) independently determined whether the outcomes were PIOs or non-PIOs, with any disagreement resolved by discussion leading to a consensus. A third author (N.M.L.) acted as an arbiter for unresolved cases. We cross-checked our selection of PIOs against that identified in the COIN initiative [24] and found our selection of clinical outcomes that were considered as PIOs to be in agreement with the outcomes identified in the project.

We report descriptive statistics and express differential proportions of PIOs according to the type of data and nature of outcomes using the risk ratio (RR) with its $95 \%$ confidence interval (CI). We examined the relationship between the year of publication of a study and the likelihood of a study having a greater number of PIOs using ordinal regression (generalized linear model option), with the number of PIOs (0 to " 5 or more") as the dependent variable and year of publication as a covariate, and express the result as the adjusted odds ratio (OR) with its 95\% CI (SPSS 22, Chicago, IL, USA).

\section{Results}

\section{General Characteristics of the Outcomes Extracted}

We extracted 6,832 outcomes from 1,882 RCTs published between 1952 and 2017 included in 276 Cochrane Neonatal reviews that were published between 1997 and 2018. There was insufficient outcome information in 8 RCTs included in 4 reviews, leaving a total of 1,874 studies for further analysis. Among these, there were 790 trials (42.1\%) with 5 or more outcomes available for extraction, and $292(15.6 \%), 315(16.8 \%), 302(16.2 \%)$, and 175 (9.3\%) with $4,3,2$, and 1 extractable outcome(s), respectively. Half of the outcomes (50.0\%) were dichotomous, the other half $(49.7 \%)$ continuous, with a small percentage $(0.3 \%)$ being ordinal. A vast majority $(92.2 \%)$ of the outcomes were objective.

Most of the outcomes (80.6\%) were determined by clinical methods alone or in combination with other modes of assessment. This was followed by laboratory parameters $(9.8 \%)$, physiological parameters $(7.1 \%)$, radiological diagnoses (1.6\%), carers'/caregivers' perception $(0.2 \%)$, and outcomes that were determined by other means (0.7\%) (Fig. 1). Most studies included a mixture of outcomes with different modes of assessment, except for 53 studies $(2.8 \%)$, of which 50 included only outcomes that were assessed predominantly clinically and 3 studies included only physiological parameters. 


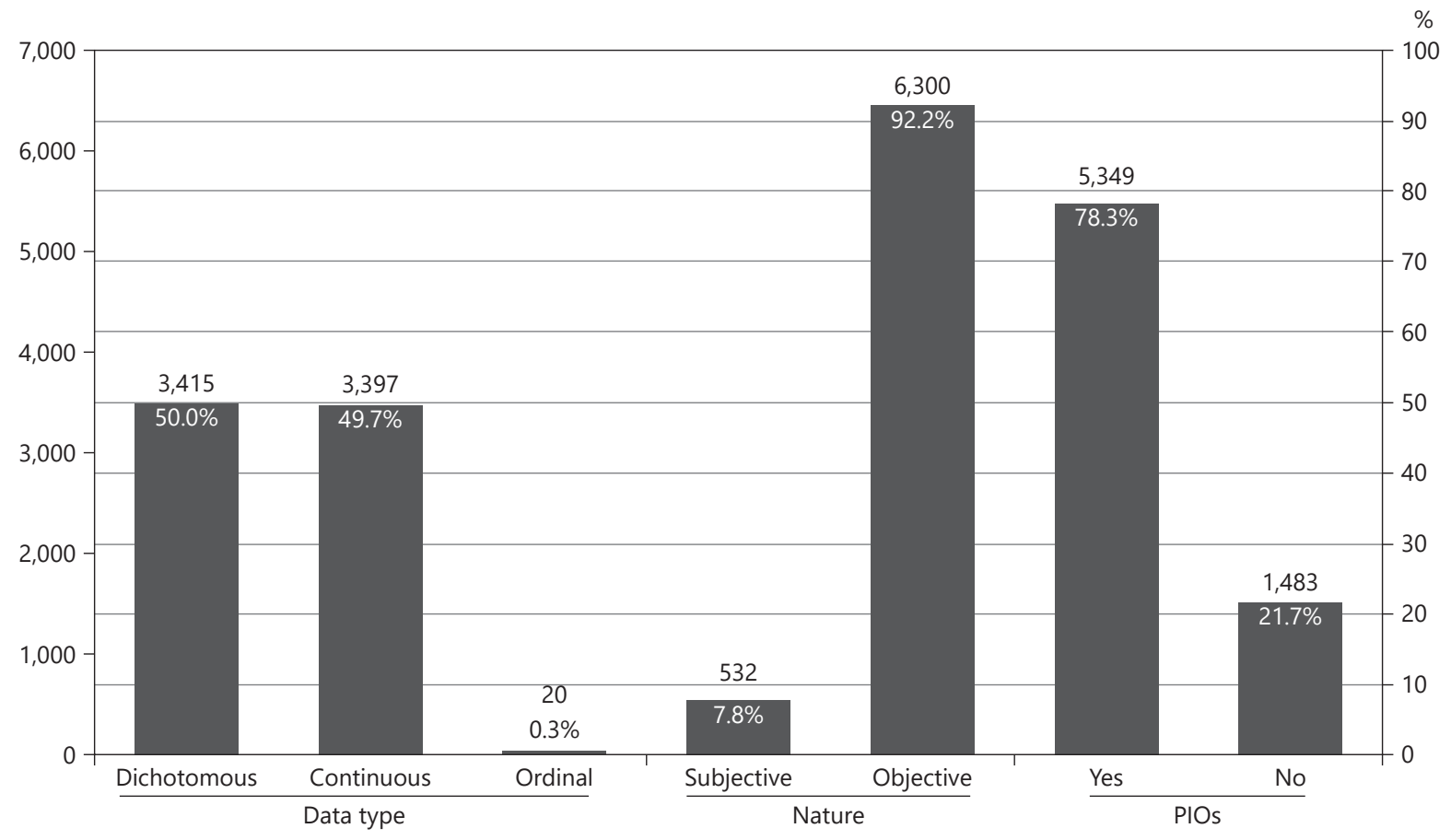

Fig. 2. Classification of outcomes according to the type of data, the nature of the outcome (subjective or objective), and whether the outcomes were considered patient-important outcomes (PIOs).

Table 1. Classification of major clinical outcomes

\begin{tabular}{lrr}
\hline Outcome & $\begin{array}{l}\text { Outcomes, } \\
n(\%)\end{array}$ & $\begin{array}{l}\text { Studies, } \\
n(\%)^{1}\end{array}$ \\
\hline Mortality/survival & $733(20.8)$ & $625(33.3)$ \\
Sepsis/infection (all types) & $331(9.4)$ & $237(12.6)$ \\
Necrotizing enterocolitis (all stages) & $217(6.2)$ & $200(10.7)$ \\
Brain injuries & $229(6.5)$ & $205(10.9)$ \\
Retinopathy of prematurity (all stages) & $120(3.4)$ & $110(5.9)$ \\
Neurodevelopmental evaluation (via all forms of evaluation & & $153(8.2)$ \\
$\quad$ and assessed in all periods) & $234(6.6)$ & $9(0.5)$ \\
Quality of life (carer/caregiver) & $12(0.3)$ & $143(7.6)$ \\
Adverse effects (all types) & $280(7.9)$ & $27(1.4)$ \\
Visual or hearing impairment & $31(0.9)$ & $171(9.1)$ \\
Bronchopulmonary dysplasia (all definitions) & $239(6.8)$ & $455(24.3)$ \\
Acute respiratory morbidities/need for respiratory support & & $352(18.8)$ \\
$\quad$ (all types) & $662(18.8)$ & $440(12.5)$ \\
\hline
\end{tabular}

Only the outcomes that are categorized in accordance with those selected in the Core Outcomes in Neonatology (COIN) initiative (displayed in the order of importance), as well as a few selected other major clinical outcomes, are displayed here. ${ }^{1}$ Percentages were calculated out of a total of 1,875 studies with outcome data. Some studies are represented multiple times, as they reported more than 1 outcome on this list. 


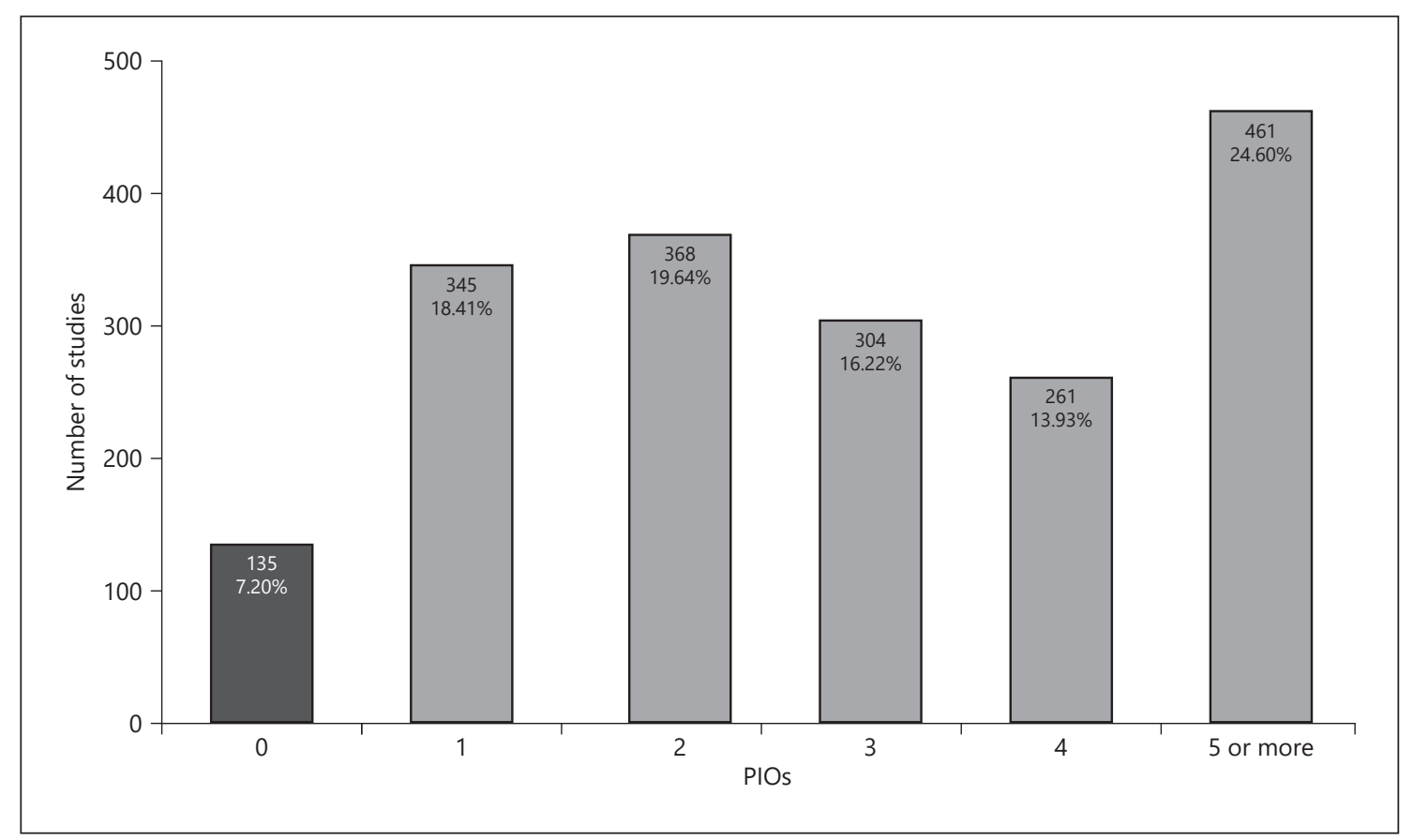

Fig. 3. Proportion of neonatal trials with 0 to " 5 or more" patient-important outcomes (PIOs) included.

A list of different outcome types, including those that were categorized under the core outcomes selected by the COIN initiative, as well as the number of studies that reported the outcomes, is shown in Table 1. As there was a wide variety in the way the study authors reported the outcomes, we categorized the outcomes broadly, without subdividing them based on further characteristics such as period and tool of evaluation.

\section{Characteristics of the PIOs}

There was fair inter-rater agreement in the initial judgement on whether the outcomes were patient important (Cohen's к: 0.69 ). A total of 5,349 outcomes (78.3\%) were considered PIOs (Fig. 2). Among the studies evaluated, $461(24.6 \%)$ included 5 PIOs or more, 261 (13.9\%) included 4 PIOs, while 1,017 (54.3\%) included 1-3 PIOs and 135 (7.2\%) did not include any PIOs (Fig. 3).

PIOs were more likely to be observed among dichotomous than among continuous outcomes (94.9 vs. $61.5 \%$; RR: 1.54 ; 95\% CI: $1.50-1.58$ ), as well as among subjective than among objective outcomes (95.9 vs. 76.8\%; RR: 1.25; 95\% CI: 1.22-1.28).

There was a small but significant association between the year of publication and the likelihood of a study having a higher number of PIOs, namely, newer studies were more likely to have a greater number of PIOs compared to older studies (adjusted OR: 1.033 with each year of publication; 95\% CI: 1.025-1.041).

\section{Discussion}

\section{Summary and Interpretation of the Main Findings}

The number of published neonatal trials is estimated at around $5 \%$ of the total number of published clinical RCTs [27]. Reassuringly, in our study, over three-quarters of the 6,832 outcomes extracted from the 1,874 RCTs were considered PIOs, and a quarter of the RCTs included 5 PIOs or more. The finding that newer studies appeared to have higher numbers of PIOs suggests an increasing awareness of trialists of the need to incorporate such outcomes in trials conducted on newborn infants. However, over 130 neonatal RCTs had no PIO included, which is a concern, as this shows that there were a significant number of studies performed on newborn infants with utilization of resources including funding, time, and expertise, but without the appropriate direct evidence to guide practice in the care of infants with similar characteristics and needs.

A higher proportion of dichotomous outcomes were considered PIOs compared to continuous outcomes, which probably reflects the fact that major clinical events, 
such as death and sepsis, are dichotomous in the form of "presence or absence," while laboratory and physiological parameters are more likely to be continuous, and scales or measurement tools that are developed to measure certain clinical outcomes or functional statuses may not capture the outcome desired accurately, especially when the instrument is not well validated, and thus may not be considered an important representation of the outcome of interest to patients $[28,29]$.

The moderate initial agreement between raters of PIOs indicated a subjective element in the judgment of the importance of an outcome. Moreover, a higher proportion of the subjective outcomes were considered PIOs compared to objective outcomes. These findings suggest that the importance placed on an outcome may vary among patients or carers/caregivers. The variation in the judgment of patient importance among outcomes emphasizes the need for outcome-related information to be presented clearly during engagements between care providers and patients or carers/caregivers, as this will enable them to prioritize the outcomes in the order of their perceived importance according to their values and preferences, as advocated in the practice of shared decision-making [30].

\section{Limitations}

We acknowledge the following limitations which might have affected the validity of our findings. First, due to time constraints, we only extracted up to 5 outcomes per trial. In trials with more than 5 outcomes, we chose the 5 outcomes that we considered the most important, which were typically the major clinical outcomes. There were 790 trials $(42.1 \%)$ with 5 or more extractable outcomes, and because of this selective limitation on the number of outcomes extracted, we were likely to have overestimated the proportion of PIOs in our study. However, we believe that the strength of our major finding, i.e., that a significant minority of the trials did not include any PIOs, was unaffected by this limitation.

We did not include patients or consumers in our identification of PIOs, but instead relied on our authors, who are either physicians or nurses, to judge the patient importance of the outcomes. The main reason for this was that in Cochrane reviews, many outcomes were described in medical terminologies that might not be understood by lay consumers, and translating them into lay language might result in a loss of accuracy. Without direct involvement of lay consumers, the PIOs selected in our study might not be entirely representative of patients' choices. We took a relatively liberal stance in our identification of PIOs, since we considered all clinical outcomes and cer- tain physiological or laboratory parameters that we deemed important in guiding patient management as PIOs, which might have led to an overestimation of PIOs. In particular, the association of some laboratory-based measurements, such as serum bilirubin and blood glucose, with corresponding major clinical complications, such as bilirubin encephalopathy and hypoglycaemia-related brain injury, respectively, remained debatable [3133]. Nonetheless, we found the majority of our clinical PIOs to be consistent with those listed in the COIN initiative [24], which were the selection of a representative group of stakeholders including patients.

Next, we relied on the information provided by the Cochrane review authors. Although the comprehensiveness and quality of the data in Cochrane reviews would have been scrutinized at the levels of the authors, editors, and peer referees against an explicit and comprehensive set of expectations [34], we did not cross-check the information against the original individual studies to verify their accuracy. We also did not cross-check the outcomes reported against the trials' published protocol or relevant information in the trial registry to assess for selective outcome reporting. This has precluded a useful assessment with regard to whether those trials with very few or no PIOs truly had no PIOs included, or whether they had selectively refrained from reporting certain important outcomes for reasons such as unappealing findings. Moreover, we were unable to determine whether the PIOs were included as primary or secondary outcomes in the studies, because many studies did not specify primary and secondary outcomes.

Furthermore, we examined outcomes only in those RCTs that were included in Cochrane Neonatal reviews. Although these are likely to represent the majority of RCTs in neonatology, we might have captured only a small proportion of the outcomes in neonatal clinical research, the majority of which are in the form of non-RCTs.

Lastly, despite having approval from Cochrane Neonatal and using all published data, we did not register our study protocol with an established trial registry. This could affect some readers' perception of the credibility of our findings.

\section{Conclusions}

The majority of the outcomes incorporated in neonatal RCTs were PIOs, and the increasing representation of PIOs in trials published over the years suggests an improving awareness by the trialists of an appropriate choice 
of outcomes in clinical trials. The recent development of a neonatal core outcome set may further improve the adoption of PIOs in neonatal trials. However, there remains the concern that a small proportion of trials had included no PIOs. Further research should explore the possible reasons for non-inclusion or non-reporting of PIOs in those trials, especially in trials where certain PIOs such as mortality and major morbidities were expected outcomes. It is also imperative to evaluate whether the PIOs in neonatal and early childhood periods, as specified in almost all the studies included here, translate into meaningful outcomes in later childhood and beyond.

\section{Disclosure Statement}

All authors have indicated they have no financial relationships relevant to this article to disclose. All authors have indicated they have no potential conflicts of interest to disclose.

\section{Funding Sources}

The study was supported by the School of Medicine, Taylor's University. There was no internal or external funding specifically for this study.

\section{References}

1 Higgins JPT, Savović J, Page MJ, Elbers RG, Sterne JAC. Chapter 8: Assessing risk of bias in a randomized trial. In: Higgins JPT, Thomas J, Chandler J, Cumpston M, Li T, Page MJ, Welch VA, editors. Cochrane Handbook for Systematic Reviews of Interventions version 6.0 [updated July 2019]. Cochrane; 2019. Available from: www.training.cochrane.org/ handbook.

2 Moher D, Hopewell S, Schulz KF, Montori V, Gøtzsche PC, Devereaux PJ, et al. CONSORT 2010 explanation and elaboration: updated guidelines for reporting parallel group randomised trials. BMJ. 2010 Mar;340:c869.

3 Webbe J, Sinha I, Gale C. Core Outcome Sets. Arch Dis Child Educ Pract Ed. 2018 Jun; 103(3):163-6.

4 Guyatt G, Oxman AD, Akl EA, Kunz R, Vist G, Brozek J, et al. GRADE guidelines: 1. Introduction - GRADE evidence profiles and summary of findings tables. J Clin Epidemiol. 2011 Apr;64(4):383-94.

$5 \mathrm{Ju}$ A, Tong A. Considerations and Challenges in Selecting Patient-Reported Outcome Measures for Clinical Trials in Nephrology. Clin J Am Soc Nephrol. 2017 Nov;12(11):1882-4.

6 Macefield RC, Boulind CE, Blazeby JM. Selecting and measuring optimal outcomes for randomised controlled trials in surgery. Langenbecks Arch Surg. 2014 Mar;399(3):26372.

7 Webbe JWH, Ali S, Sakonidou S, Webbe T, Duffy JMN, Brunton G, et al. Inconsistent outcome reporting in large neonatal trials: a systematic review. Arch Dis Child Educ Pract Ed. 2020 Jan;105(1):69-75.

8 Williamson PR, Altman DG, Blazeby JM, Clarke M, Devane D, Gargon E, et al. Developing core outcome sets for clinical trials: issues to consider. Trials. 2012 Aug; 13(1):132.

9 Clarke M. Standardising outcomes for clinical trials and systematic reviews. Trials. 2007 Nov;8(1):39.
10 Curtis L, Hernandez AF, Weinfurt KP. Choosing and Specifying Endpoints and Outcomes: Meaningful Endpoints. 2019. In: Rethinking Clinical Trials: A Living Textbook of Pragmatic Clinical Trials [Internet]. Bethesda, MD: NIH Health Care Systems Research Collaboratory. Available from: https://rethinkingclinicaltrials.org/chapters/design/ choosing-specifying-end-points-outcomes/ meaningful-endpoints/.

11 Sinha IP, Altman DG, Beresford MW, Boers M, Clarke M, Craig J, et al.; StaR Child Health Group. Standard 5: selection, measurement, and reporting of outcomes in clinical trials in children. Pediatrics. 2012 Jun;129 Suppl 3: S146-52.

12 Fleming TR, Powers JH. Biomarkers and surrogate endpoints in clinical trials. Stat Med. 2012 Nov;31(25):2973-84.

13 Twaddell S. Surrogate outcome markers in research and clinical practice. Aust Prescr. 2009; 32(2):47-50.

14 Montori VM, Isley WL, Guyatt GH. Waking up from the DREAM of preventing diabetes with drugs. BMJ. 2007 Apr;334(7599):882-4.

15 Fleming TR, DeMets DL. Surrogate end points in clinical trials: are we being misled? Ann Intern Med. 1996 Oct;125(7):605-13.

16 Guyatt G, Montori V, Devereaux PJ, Schünemann $\mathrm{H}$, Bhandari M. Patients at the centre: in our practice, and in our use of language. Evid Based Med. 2004;9(1):6-7.

17 Møller MH. Patient-important outcomes and core outcome sets: increased attention needed! Br J Anaesth. 2019 Apr;122(4):408-10.

18 Yordanov Y, Dechartres A, Ravaud P. Patient-important outcomes in systematic reviews: poor quality of evidence. PLoS One. 2018 Apr;13(4):e0195460.

19 Prinsen CA, Vohra S, Rose MR, Boers M, Tugwell P, Clarke M, et al. How to select outcome measurement instruments for outcomes included in a "Core Outcome Set" - a practical guideline. Trials. 2016 Sep;17(1): 449.
20 Williamson PR, Altman DG, Bagley H, Barnes KL, Blazeby JM, Brookes ST, et al. The COMET Handbook: version 1.0. Trials. 2017 Jun; 18(3 Suppl 3):280.

21 Bagley HG, Morley R, Young B. Using patient-important outcomes for systematic reviews. Oxford Cochrane UK; 2017. Available from: http://gdt.guidelinedevelopment.org/ app/handbook/handbook.html\#h. li2bwkm8zpjo.

22 Meyer S, Butte M, Gortner L. Research in neonatology: a global perspective. Acta Paediatr. 2013 Aug; 102(8):e346.

23 Modi N. Neonatal research. Lancet. 1998 Feb; 351(9101):530.

24 Webbe JWH, Duffy JMN, Afonso E, Al-Muzaffar I, Brunton G, Greenough A, et al. Core outcomes in neonatology: development of a core outcome set for neonatal research. Arch Dis Child Fetal Neonatal Ed. 2019 [Epub ahead of print].

25 Dyson MP, Shave K, Gates A, Fernandes RM, Scott SD, Hartling L. Which outcomes are important to patients and families who have experienced paediatric acute respiratory illness? Findings from a mixed methods sequential exploratory study. BMJ Open. 2017 Dec; 7(12): 018199.

26 The GRADE Working Group. Chapter 3. Selecting and rating the importance of outcomes. McMaster University; 2013. Available from: http:/gdt.guidelinedevelopment.org/ app/handbook/handbook.html\#h. li2bwkm8zpjo.

27 How many randomized trials are published each year? TrustTheEvidence.net; 2010. Available from: http://blogs.trusttheevidence. net/carl-heneghan/how-many-randomizedtrials-are-published-each-year.

28 Coster WJ. Making the best match: selecting outcome measures for clinical trials and outcome studies. Am J Occup Ther. 2013;67(2): 162-70. 
29 Velentgas P, Dreyer NA, Wu AW. Chapter 6: Outcome Definition and Measurement. In: Velentgas P, Dreyer NA, Nourjah P, et al., editors. Developing a Protocol for Observational Comparative Effectiveness Research: A User's Guide. Rockville (MD): Agency for Healthcare Research and Quality (US); 2013.

30 Stiggelbout AM, Van der Weijden T, De Wit MP, Frosch D, Légaré F, Montori VM, et al. Shared decision making: really putting patients at the centre of healthcare. BMJ. 2012 Jan;344:e256.
31 Ip S, Chung M, Kulig J, O’Brien R, Sege R, Glicken S, et al.; American Academy of Pediatrics Subcommittee on Hyperbilirubinemia. An evidence-based review of important issues concerning neonatal hyperbilirubinemia. Pediatrics. 2004 Jul;114(1):e130-53.

32 Gerard JP, Ngim CF. How strong is serum bilirubin a predictor of bilirubin-induced neurologic dysfunction (BIND) in neonates: a systematic review of correlational studies. 3rd KL International Neonatology Conference Kuala Lumpur, Malaysia: The University of Malaya, Perinatal Society of Malaysia, College of Paediatrics, Academy of Medicine, Malaysia; 2019
33 Cornblath M, Hawdon JM, Williams AF, Aynsley-Green A, Ward-Platt MP, Schwartz $\mathrm{R}$, et al. Controversies regarding definition of neonatal hypoglycemia: suggested operational thresholds. Pediatrics. 2000 May; 105(5): 1141-5.

34 Churchill RL, Chandler J, Tovey D, Thomas J, Flemyng E, Higgins JP. Standards for the reporting of new Cochrane Intervention Reviews. Methodological Expectations of Cochrane Intervention Reviews [Internet]. London: Cochrane; 2019 October. 